\title{
Lifting Artin-Schreier covers with maximal wild monodromy
}

\author{
P. Chrétien
}

October 2, 2017

\begin{abstract}
Let $k$ be an algebraically closed field of characteristic $p>0$. We consider the problem of lifting $p$-cyclic covers of $\mathbb{P}_{k}^{1}$ as $p$-cyclic covers of the projective line over some DVR under the condition that the wild monodromy is maximal. We answer positively the question for covers birational to $w^{p}-w=t R(t)$ for some additive polynomial $R(t)$.
\end{abstract}

\section{Introduction}

Let $(R, v)$ be a complete discrete valuation ring of mixed characteristic $(0, p)$ with fraction field $K$ containing a primitve $p$-th root of unity $\zeta_{p}$ and algebraically closed residue field $k$. The stable reduction theorem states that given a smooth, projective, geometrically connected curve $C / K$ of genus $g(C) \geq 2$, there exists a unique minimal Galois extension $M / K$ called the monodromy extension of $C / K$ such that $C_{M}:=C \times M$ has stable reduction over $M$. The group $G=\operatorname{Gal}(M / K)$ is the monodromy group of $C / K$.

Let us consider the case where $\phi: C \rightarrow \mathbb{P}_{K}^{1}$ is a $p$-cyclic cover. Let $\mathcal{C}$ be the stable model of $C_{M} / M$ and $\operatorname{Aut}_{k}\left(\mathcal{C}_{k}\right)^{\#}$ be the subgroup of $\operatorname{Aut}_{k}\left(\mathcal{C}_{k}\right)$ of elements acting trivially on the reduction in $\mathcal{C}_{k}$ of the ramification locus of $\phi \times \operatorname{Id}_{M}: C_{M} \rightarrow \mathbb{P}_{M}^{1}$ (see [Liu02] 10.1.3 for the definition of the reduction map of $C_{M}$ ). One derives from the stable reduction theorem the following injection

$$
\operatorname{Gal}(M / K) \hookrightarrow \operatorname{Aut}_{k}\left(\mathcal{C}_{k}\right)^{\#} .
$$

When the $p$-Sylow subgroups of these groups are isomorphic, one says that the wild monodromy is maximal. We are interested in realization of smooth covers as above such that the $p$-adic valuation of $\left|\operatorname{Aut}_{k}\left(\mathcal{C}_{k}\right)^{\#}\right|$ is large compared to the genus of $\mathcal{C}_{k}$ and having maximal wild monodromy. Moreover, 
we will study the ramification filtration and the Swan conductor of their monodromy extension.

Recall that a big action is a pair $(X, G)$ where $X / k$ is a smooth, projective, geometrically connected curve of genus $g(X) \geq 2$ and $G$ is a finite $p$-group of $k$-automorphisms of $X / k$ such that $|G|>\frac{2 p}{p-1} g(X)$. According to LM05] Theorem 1.1 II f), if $(X, G)$ is a big action, then one has that $|G| \leq \frac{4 p}{(p-1)^{2}} g(X)^{2}$ with equality if and only if $X / k$ is birationally given by $w^{p}-w=t R(t)$ where $R(t) \in k[t]$ is an additive polynomial. In this case, $G$ is an extra-special $p$-group and equals the $p$-Sylow subgroup $G_{\infty, 1}(X)$ of the subgroup of $\operatorname{Aut}_{k}(X)$ leaving $t=\infty$ fixed.

This motivates the following question, with the above notations, given a big action $(C, G)$ such that $|G|=\frac{4 p}{(p-1)^{2}} g(X)^{2}$, is it possible to find a field $K$ and a a $p$-cyclic cover $C / K$ of $\mathbb{P}_{K}^{1}$ such that $\mathcal{C}_{k} \simeq X$, that $G \simeq \operatorname{Aut}\left(\mathcal{C}_{k}\right)_{1}^{\#}$ is a $p$-Sylow subgroup of $\operatorname{Aut}\left(\mathcal{C}_{k}\right)^{\#}$ and the curve $C / K$ has maximal wild monodromy?

Let $n \in \mathbb{N}^{\times}, q=p^{n}, \lambda=\zeta_{p}-1$ and $K=\mathbb{Q}_{p}^{\text {ur }}\left(\lambda^{1 /(1+q)}\right)$. For any additive polynomial $R(t) \in k[t]$ of degree $q$, let $X / k$ be curve defined by $w^{p}-w=t R(t)$. In section 3, we prove the following

Theorem 1.1. There exists a p-cyclic cover $C / K$ of $\mathbb{P}_{K}^{1}$ such that $\mathcal{C}_{k} \simeq X$, one has $G_{\infty, 1}(X) \simeq \operatorname{Aut}\left(\mathcal{C}_{k}\right)_{1}^{\#}$ and the curve $C / K$ has maximal wild monodromy $M / K$. The extension $M / K$ is the decomposition field of an explicitly given polynomial and the group $\operatorname{Gal}(M / K) \simeq \operatorname{Aut}_{k}\left(\mathcal{C}_{k}\right)_{1}^{\#}$ is an extra-special p-group of order $p q^{2}$.

The group $G_{\infty, 1}\left(\mathcal{C}_{k}\right)=\operatorname{Aut}_{k}\left(\mathcal{C}_{k}\right)_{1}^{\#}$ is endowed with the ramification filtration $\left(G_{\infty, i}\left(\mathcal{C}_{k}\right)\right)_{i \geq 0}$ which is easily seen to be :

$$
G_{\infty, 0}\left(\mathcal{C}_{k}\right)=G_{\infty, 1}\left(\mathcal{C}_{k}\right) \supsetneq \mathrm{Z}\left(G_{\infty, 0}\left(\mathcal{C}_{k}\right)\right)=G_{\infty, 2}\left(\mathcal{C}_{k}\right)=\cdots=G_{\infty, 1+q}\left(\mathcal{C}_{k}\right) \supsetneq\{1\} .
$$

Moreover, $G:=\operatorname{Gal}(M / K)$ being the Galois group of a finite extension of $K$, it is endowed with the ramification filtration $\left(G_{i}\right)_{i \geq 0}$. Since $G \simeq G_{\infty, 1}\left(\mathcal{C}_{k}\right)$ it is natural to ask for the behaviour of $\left(G_{i}\right)_{i \geq 0}$ under (1), that is to compare $\left(G_{i}\right)_{i \geq 0}$ and $\left(G_{\infty, i}\left(\mathcal{C}_{k}\right)\right)_{i \geq 0}$. In the general case, the arithmetic is quite tedious due to the expression of the lifting of $X / k$. Actually we could not obtain a numerical example for the easiest case when $p=3$. Nonetheless, when $p=2$, one computes the conductor exponent $f(\operatorname{Jac}(C) / K)$ of $\operatorname{Jac}(C) / K$ and its Swan conductor $\operatorname{sw}(\operatorname{Jac}(C) / K)$ :

Theorem 1.2. Under the hypotheses of Theorem 1.1, if $p=2$ the lower ramification filtration of $G$ is :

$$
G=G_{0}=G_{1} \supsetneq \mathrm{Z}(G)=G_{2}=\cdots=G_{1+q} \supsetneq\{1\} .
$$


Then, $f(\operatorname{Jac}(C) / K)=2 q+1$ and $\operatorname{sw}\left(\operatorname{Jac}(C) / \mathbb{Q}_{2}^{\text {ur }}\right)=1$.

\section{Remarks :}

1. In Theorem 1.1, one actually obtains a family of liftings $C / K$ of $X / k$ with the announced properties. It is worth noting that there are finitely many additive polynomials $R_{0}(t) \in k[t]$ such that $w^{p}-w=t R(t)$ is $k$ isomorphic to $w^{p}-w=t R_{0}(t)$ (see [LM05] 8.2), so we have to solve the problem in a somehow generic way. In CM11, we obtain the analogous of Theorem 1.1 and Theorem 1.2 for $p \geq 2$ in the easier case $R(t)=t^{q}$.

2. For $p=3$, the easiest non-trivial case is such that $[M: K]=243$, that is why we could not even do computations using Magma to guess the behaviour of the ramification filtration of the monodromy extension for $p>2$. Nonetheless, one shows that if $p \geq 3$, the lower ramification filtration of $G$ is

$$
G=G_{0}=G_{1} \supsetneq G_{2}=\cdots=G_{u}=\mathrm{Z}(G) \supsetneq\{1\},
$$

where $u \in 1+q \mathbb{N}$.

3. The value $\operatorname{sw}\left(\operatorname{Jac}(C) / \mathbb{Q}_{2}^{\text {ur }}\right)=1$ is the smallest one among abelian varieties over $\mathbb{Q}_{2}^{u r}$ with non tame monodromy extension. That is, in some sense, a counter part of [BK05] and [LRS93] where an upper bound for the conductor exponent is given and it is shown that this bound is actually achieved.

\section{Background}

Notations. Let $(R, v)$ be a complete discrete valuation ring (DVR) of mixed characteristic $(0, p)$ with fraction field $K$ and algebraically closed residue field $k$. We denote by $\pi_{K}$ a uniformizer of $R$ and assume that $K$ contains a primitive $p$-th root of unity $\zeta_{p}$. Let $\lambda:=\zeta_{p}-1$. If $L / K$ is an algebraic extension, we will denote by $\pi_{L}$ (resp. $v_{L}$, resp. $L^{\circ}$ ) a uniformizer for $L$ (resp. the prolongation of $v$ to $L$ such that $v_{L}\left(\pi_{L}\right)=1$, resp. the ring of integers of $L$ ). If there is no possible confusion we note $v$ for the prolongation of $v$ to an algebraic closure $K^{\text {alg }}$ of $K$.

1. Stable reduction of curves. The first result is due to Deligne and Mumford (see for example Liu02 for a presentation following Artin and Winters). 
Theorem 2.1 (Stable reduction theorem). Let $C / K$ be a smooth, projective, geometrically connected curve over $K$ of genus $g(C) \geq 2$. There exists a unique finite Galois extension $M / K$ minimal for the inclusion relation such that $C_{M} / M$ has stable reduction. The stable model $\mathcal{C}$ of $C_{M} / M$ over $M^{\circ}$ is unique up to isomorphism. One has a canonical injective morphism :

$$
\operatorname{Gal}(M / K) \stackrel{i}{\hookrightarrow} \operatorname{Aut}_{k}\left(\mathcal{C}_{k}\right)
$$

\section{Remarks :}

1. Let's explain the action of $\operatorname{Gal}\left(K^{\text {alg }} / K\right)$ on $\mathcal{C}_{k} / k$. The group $\operatorname{Gal}\left(K^{\text {alg }} / K\right)$ acts on $C_{M}:=C \times M$ on the right. By unicity of the stable model, this action extends to $\mathcal{C}$ :

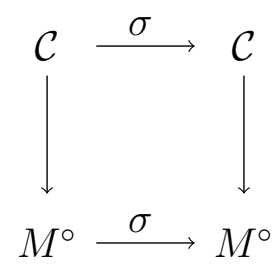

Since $k=k^{\text {alg }}$ one gets $\sigma \times k=\operatorname{Id}_{k}$, whence the announced action. The last assertion of the theorem characterizes the elements of $\mathrm{Gal}\left(K^{\mathrm{alg}} / M\right)$ as the elements of $\operatorname{Gal}\left(K^{\text {alg }} / K\right)$ that trivially act on $\mathcal{C}_{k} / k$.

2. If $p>2 g(C)+1$, then $C / K$ has stable reduction over a tamely ramified extension of $K$. We will study examples of covers with $p \leq 2 g(C)+1$.

3. Our results will cover the elliptic case. Let $E / K$ be an elliptic curve with additive reduction. If its modular invariant is integral, then there exists a smallest extension $M$ of $K$ over which $E / K$ has good reduction. Else $E / K$ obtains split multiplicative reduction over a unique quadratic extension of $K$ ( see [Kra90]).

Definition 2.1. The extension $M / K$ is the monodromy extension of $C / K$. We call $\operatorname{Gal}(M / K)$ the monodromy group of $C / K$. It has a unique $p$ Sylow subgroup $\operatorname{Gal}(M / K)_{1}$ called the wild monodromy group. The extension $M / M^{\operatorname{Gal}(M / K)_{1}}$ is the wild monodromy extension.

From now on we consider smooth, projective, geometrically integral curves $C / K$ of genus $g(C) \geq 2$ birationally given by $Y^{p}=f(X):=\prod_{i=0}^{t}\left(X-x_{i}\right)^{n_{i}}$ with $\left(p, \sum_{i=0}^{t} n_{i}\right)=1,\left(p, n_{i}\right)=1$ and $\forall 0 \leq i \leq t, x_{i} \in R^{\times}$. Moreover, we assume that $\forall i \neq j, v\left(x_{i}-x_{j}\right)=0$, that is to say, the branch locus 
$B=\left\{x_{0}, \ldots, x_{t}, \infty\right\}$ of the cover has equidistant geometry. We denote by Ram the ramification locus of the cover.

Remark : We only ask p-cyclic covers to satisfy Raynaud's theorem 1' Ray90 condition, that is the branch locus is $K$-rational with equidistant geometry. This has consequences on the image of (2).

Proposition 2.1. Let $\mathcal{T}=\operatorname{Proj}\left(M^{\circ}\left[X_{0}, X_{1}\right]\right)$ with $X=X_{0} / X_{1}$. The normalization $\mathcal{Y}$ of $\mathcal{T}$ in $K\left(C_{M}\right)$ admits a blowing-up $\tilde{\mathcal{Y}}$ which is a semi-stable model of $C_{M} / M$. The dual graph of $\tilde{\mathcal{Y}}_{k} / k$ is a tree and the points in Ram specialize in a unique irreducible component $D_{0} \simeq \mathbb{P}_{k}^{1}$ of $\tilde{\mathcal{Y}}_{k} / k$. There exists a contraction morphism $h: \tilde{\mathcal{Y}} \rightarrow \mathcal{C}$, where $\mathcal{C}$ is the stable model of $C_{M} / M$ and

$$
\operatorname{Gal}(M / K) \hookrightarrow \operatorname{Aut}_{k}\left(\mathcal{C}_{k}\right)^{\#},
$$

where $\operatorname{Aut}_{k}\left(\mathcal{C}_{k}\right)^{\#}$ is the subgroup of $\operatorname{Aut}_{k}\left(\mathcal{C}_{k}\right)$ of elements inducing the identity on $h\left(D_{0}\right)$.

Proof. see [CM11].

Remark : The component $D_{0}$ is the so called original component.

Definition 2.2. If (3) is surjective, we say that $C$ has maximal monodromy. If $v_{p}(|\operatorname{Gal}(M / K)|)=v_{p}\left(\left|\operatorname{Aut}_{k}\left(\mathcal{C}_{k}\right)^{\#}\right|\right)$, we say that $C$ has maximal wild monodromy.

Definition 2.3. The valuation on $K(X)$ corresponding to the discrete valuation ring $R[X]_{\left(\pi_{K}\right)}$ is called the Gauss valuation $v_{X}$ with respect to $X$. We then have

$$
v_{X}\left(\sum_{i=0}^{m} a_{i} X^{i}\right)=\min \left\{v\left(a_{i}\right), 0 \leq i \leq m\right\} .
$$

Note that a change of variables $T=\frac{X-y}{\rho}$ for $y, \rho \in R$ induces a Gauss valuation $v_{T}$. These valuations are exactly those that come from the local rings at generic points of components in the semi-stables models of $\mathbb{P}_{K}^{1}$.

2. Extra-special p-groups. The Galois groups and automorphism groups that we will have to consider are $p$-groups with peculiar group theoretic properties (see for example Hup67 Kapitel III $\S 13$ or [Suz86] for an account on extra-special $p$-groups). We will denote by $\mathrm{Z}(G)$ (resp. $\mathrm{D}(G), \Phi(G)$ ) the center (resp. the derived subgroup, the Frattini subgroup) of $G$. If $G$ is a p-group, one has $\Phi(G)=\mathrm{D}(G) G^{p}$. 
Definition 2.4. An extra-special p-group is a non abelian p-group $G$ such that $\mathrm{D}(G)=\mathrm{Z}(G)=\Phi(G)$ has order $p$.

Proposition 2.2. Let $G$ be an extra-special p-group.

1. Then $|G|=p^{2 n+1}$ for some $n \in \mathbb{N}^{\times}$.

2. One has the exact sequence

$$
0 \rightarrow \mathrm{Z}(G) \rightarrow G \rightarrow(\mathbb{Z} / p \mathbb{Z})^{2 n} \rightarrow 0
$$

3. The group $G$ has an abelian subgroup $J$ such that $\mathrm{Z}(G) \subseteq J$ and $|J / \mathrm{Z}(G)|=p^{n}$.

3. Galois extensions of complete DVRs. Let $L / K$ be a finite Galois extension with group $G$. Then $G$ is endowed with a lower ramification filtration $\left(G_{i}\right)_{i \geq-1}$ where $G_{i}$ is the $i$-th lower ramification group defined by $G_{i}:=\left\{\sigma \in G \mid v_{L}\left(\sigma\left(\pi_{L}\right)-\pi_{L}\right) \geq i+1\right\}$. The integers $i$ such that $G_{i} \neq G_{i+1}$ are called lower breaks. For $\sigma \in G-\{1\}$, let $i_{G}(\sigma):=v_{L}\left(\sigma\left(\pi_{L}\right)-\pi_{L}\right)$. The group $G$ is also endowed with a higher ramification filtration $\left(G^{i}\right)_{i \geq-1}$ which can be computed from the $G_{i}$ 's by means of the Herbrand's function $\varphi_{L / K}$. The real numbers $t$ such that $\forall \epsilon>0, G^{t+\epsilon} \neq G^{t}$ are called higher breaks.

Lemma 2.1. Let $M / K$ be a Galois extension such that $\operatorname{Gal}(M / K)$ is an extra-special p-group of order $p^{2 n+1}$. Assume that $\operatorname{Gal}\left(M^{\mathrm{Z}(G)} / K\right)_{2}=\{1\}$, then the break $t$ of $M / M^{\mathrm{Z}(G)}$ is such that $t \in 1+p^{n} \mathbb{N}$.

Proof. According to Proposition 2.2 3., there exists an abelian subgroup $J$ with $\mathrm{Z}(G) \subseteq J \subseteq G$ and $|J / \mathrm{Z}(G)|=p^{n}$. Thus, one has the following diagram

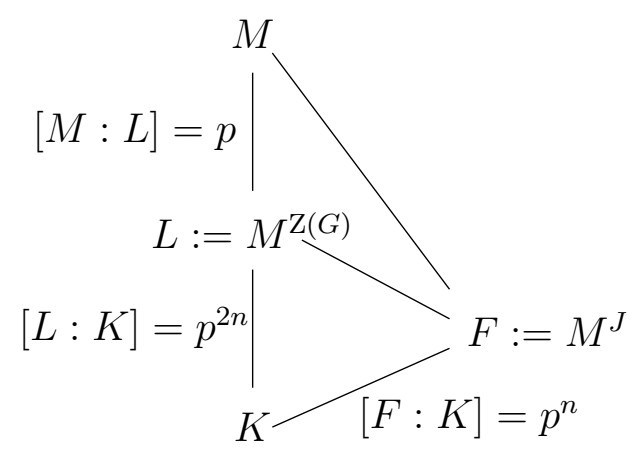

Let $t$ be the lower break of $M / L$, then $t$ is a lower break of $M / F$ and $\varphi_{M / F}(t)=\varphi_{L / F}\left(\varphi_{M / L}(t)\right)$ is a higher break of $M / F$. Since $\varphi_{M / L}(t)=t$, one 
has $\varphi_{M / F}(t)=\varphi_{L / F}(t)$. Since $\operatorname{Gal}(L / K)_{2}=\{1\}$, one has $\operatorname{Gal}(L / F)_{2}=\{1\}$ and $\varphi_{L / F}(t)=1+\frac{t-1}{p^{n}}$. The Hasse-Arf Theorem applied to the abelian extension $M / F$ implies that $1+\frac{t-1}{p^{n}} \in \mathbb{N}-\{0\}$, thus $t \in 1+p^{n} \mathbb{N}$.

4. Torsion points on abelian varieties. Let $A / K$ be an abelian variety over $K$ with potential good reduction and $\ell \neq p$ be a prime number. We denote by $A[\ell]$ the $\ell$-torsion group of $A\left(K^{\text {alg }}\right)$ and by $T_{\ell}(A)=\lim A\left[\ell^{n}\right]$ (resp. $\left.V_{\ell}(A)=T_{\ell}(A) \otimes \mathbb{Q}_{\ell}\right)$ the Tate module (resp. $\ell$-adic Tate module) of $A$.

The following result may be found in Gur03 (paragraph 3). We recall it for the convenience of the reader.

Lemma 2.2. Let $k=k^{\text {alg }}$ be a field with char $k=p \geq 0$ and $C / k$ be a projective, smooth, integral curve. Let $\ell \neq p$ be a prime number and $H$ be a finite subgroup of $\operatorname{Aut}_{k}(C)$ such that $(|H|, \ell)=1$. Then

$$
2 g(C / H)=\operatorname{dim}_{\mathbb{F}_{\ell}} \operatorname{Jac}(C)[\ell]^{H} .
$$

If $\ell \geq 3$, then $L=K(A[\ell])$ is the minimal extension over which $A / K$ has good reduction. It is a Galois extension with group $G$ (see [ST68]). We denote by $r_{G}$ (resp. $1_{G}$ ) the character of the regular (resp. unit) representation of $G$. We denote by $I$ the inertia group of $K^{\text {alg }} / K$. For further explanations about conductor exponents see [Ser67], Ogg67] and [ST68].

Definition 2.5. 1. Let

$$
\begin{aligned}
& a_{G}(\sigma):=-i_{G}(\sigma), \quad \sigma \neq 1, \\
& a_{G}(1):=\sum_{\sigma \neq 1} i_{G}(\sigma),
\end{aligned}
$$

and $\mathrm{sw}_{G}:=a_{G}-r_{G}+1_{G}$. Then, $a_{G}$ is the character of $a \mathbb{Q}_{\ell}[G]$-module and there exists a projective $\mathbb{Z}_{\ell}[G]$-module $\mathrm{Sw}_{G}$ such that $\mathrm{Sw}_{G} \otimes_{\mathbb{Z}_{\ell}} \mathbb{Q}_{\ell}$ has character $\mathrm{Sw}_{G}$.

2. We still denote by $T_{\ell}(A)$ (resp. $A[\ell]$ ) the $\mathbb{Z}_{\ell}[G]$-module (resp. $\mathbb{F}_{\ell}[G]$ module) afforded by $G \rightarrow \operatorname{Aut}\left(T_{\ell}(A)\right)$ (resp. $\left.G \rightarrow \operatorname{Aut}(A[\ell])\right)$. Let

$$
\begin{aligned}
\mathrm{sw}(A / K) & :=\operatorname{dim}_{\mathbb{F}_{\ell}} \operatorname{Hom}_{G}\left(\operatorname{Sw}_{G}, A[\ell]\right), \\
\epsilon(A / K) & :=\operatorname{codim}_{\mathbb{Q}_{\ell}} V_{\ell}(A)^{I} .
\end{aligned}
$$

The integer $f(A / K):=\epsilon(A / K)+\mathrm{sw}(A / K)$ is the so called conductor exponent of $A / K$ and $\operatorname{sw}(A / K)$ is the Swan conductor of $A / K$. 
Proposition 2.3. Let $\ell \neq p, \ell \geq 3$ be a prime number.

1. The integers $\operatorname{sw}(A / K)$ and $\epsilon(A / K)$ are independent of $\ell$.

2. One has

$$
\operatorname{sw}(A / K)=\sum_{i \geq 1} \frac{\left|G_{i}\right|}{\left|G_{0}\right|} \operatorname{dim}_{\mathbb{F}_{\ell}} A[\ell] / A[\ell]^{G_{i}} .
$$

Moreover, for $\ell$ large enough, $\epsilon(A / K)=\operatorname{dim}_{\mathbb{F}_{\ell}} A[\ell] / A[\ell]^{G_{0}}$.

Remark : It follows from the definition that $\operatorname{sw}(A / K)=0$ if and only if $G_{1}=\{1\}$. The Swan conductor is a measure of the wild ramification.

5. Automorphisms of Artin-Schreier covers. See [LM05] for further results on this topic. Let $R(t) \in k[t]$ be a monic additive polynomial and $A_{R} / k$ be the smooth, projective, geometrically irreducible curve birationally given by $w^{p}-w=t R(t)$. There is a so called Artin-Schreier morphism $\pi: A_{R} \rightarrow \mathbb{P}_{k}^{1}$. The automorphism $t_{a}$ of $\mathbb{P}_{k}^{1}$ given by $t \mapsto t+a$ with $a \in k$ has a prolongation $\tilde{t}_{a}$ to $A_{R}$ if there is a commutative diagram

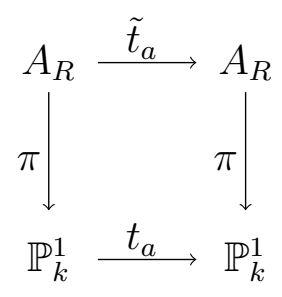

Proposition 2.4. Let $n \geq 1, q:=p^{n}$ and $R(t):=\sum_{k=0}^{n-1} \bar{u}_{k} t^{p^{k}}+t^{q} \in k[t]$. The automorphism of $\mathbb{P}_{k}^{1}$ given by $t \mapsto t+a$ with $a \in k$ has a prolongation to $A_{R} / k$ if and only if one has

$$
a^{q^{2}}+\left(2 \bar{u}_{0} a\right)^{q}+\sum_{k=1}^{n-1}\left(\bar{u}_{k}^{q} a^{q p^{k}}+\left(\bar{u}_{k} a\right)^{q / p^{k}}\right)+a=0 .
$$

\section{Main theorem}

We start by fixing notations that will be used throughout this section.

Notations. We denote by $\mathfrak{m}$ the maximal ideal of $\left(K^{\text {alg }}\right)^{\circ}$. Let $n \in \mathbb{N}^{\times}$, $q:=p^{n}, a_{n}:=(-1)^{q}(-p)^{p+p^{2}+\cdots+q}$ and $\forall 0 \leq i \leq n-1, d_{i}:=p^{n-i+1}+\cdots+q$. We denote by $\mathbb{Q}_{p}^{\text {ur }}$ the maximal unramified extension of $\mathbb{Q}_{p}$ and we put 
$K:=\mathbb{Q}_{p}^{\text {ur }}\left(\lambda^{1 /(1+q)}\right)$. Let $\underline{\rho}:=\left(\rho_{0}, \ldots, \rho_{n-1}\right)$ where $\forall 0 \leq k \leq n-1, \rho_{k} \in K$, $\rho_{k}=u_{k} \lambda^{p\left(q-p^{k}\right) /(1+q)}$ and $v\left(u_{k}\right)=0$ or $u_{k}=0$. For $c \in R$, let

$$
\begin{aligned}
& f_{c, \rho}(X):=1+\sum_{k=0}^{n-1} \rho_{k} X^{1+p^{k}}+c X^{q}+X^{1+q}, \\
& \text { and } s_{1, \rho}(X):=2 \rho_{0} X+\sum_{k=1}^{n-1} \rho_{k} X^{p^{k}}+X^{q} .
\end{aligned}
$$

One defines the modified monodromy polynomial $L_{c, \rho}(X)$ by $s_{1, \rho}(X)^{q}-a_{n} f_{c, \rho}(X)^{q-1}(c+X)-(-1)^{q} \sum_{k=1}^{n-1}\left(\rho_{k} X\right)^{q / p^{k}}(-p)^{d_{k}} f_{c, \rho}(X)^{q\left(p^{k}-1\right) / p^{k}}$.

Let $C_{c, \rho} / K$ and $A_{u} / k$ be the smooth projective integral curves birationally given respectively by $Y^{p}=f_{c, \rho}(X)$ and $w^{p}-w=\sum_{k=0}^{n-1} \bar{u}_{k} t^{1+p^{k}}+t^{1+q}$.

Theorem 3.1. The curve $C_{c, \rho} / K$ has potential good reduction isomorphic to $A_{u} / k$.

1. If $v(c) \geq v\left(\lambda^{p /(1+q)}\right)$, then the monodromy extension of $C_{c, \rho} / K$ is trivial.

2. If $v(c)<v\left(\lambda^{p /(1+q)}\right)$, let $y$ be a root of $L_{c, \rho}(X)$ in $K^{\text {alg }}$. Then $C_{c, \rho}$ has good reduction over $K\left(y, f_{c, \rho}(y)^{1 / p}\right)$. If $L_{c, \rho}(X)$ is irreducible over $K$, then $C_{c, \rho} / K$ has maximal wild monodromy. The monodromy extension of $C_{c, \rho} / K$ is $M=K\left(y, f_{c, \rho}(y)^{1 / p}\right)$ and $G=\operatorname{Gal}(M / K)$ is an extraspecial p-group of order $p q^{2}$.

3. Assume that $c=1$. The polynomial $L_{1, \rho}(X)$ is irreducible over $K$. The lower ramification filtration of $G$ is

$$
G=G_{0}=G_{1} \supsetneq G_{2}=\cdots=G_{u}=\mathrm{Z}(G) \supsetneq\{1\},
$$

with $u \in 1+q \mathbb{N}$. Moreover, if $p=2$, then $u=1+q$, one has $f\left(\operatorname{Jac}\left(C_{1, \rho}\right) / K\right)=2 q+1$ and $\operatorname{sw}\left(\operatorname{Jac}\left(C_{1, \rho}\right) / \mathbb{Q}_{2}^{\text {ur }}\right)=1$.

Proof. 1. Assume that $v(c) \geq v\left(\lambda^{p /(1+q)}\right)$. Set $\lambda^{p /(1+q)} T=X$ and $\lambda W+1=Y$. Then, the equation defining $C_{c, \rho} / K$ becomes

$$
(\lambda W+1)^{p}=1+\sum_{k=0}^{n-1} \rho_{k} \lambda^{p\left(1+p^{k}\right) /(1+q)} T^{1+p^{k}}+c \lambda^{p q /(1+q)} T^{q}+\lambda^{p} T^{1+q} .
$$


After simplification by $\lambda^{p}$ and reduction modulo $\pi_{K}$ this equation gives :

$$
w^{p}-w=\sum_{k=0}^{n-1} \bar{u}_{k} t^{1+p^{k}}+a t^{q}+t^{1+q}, a \in k .
$$

By Hurwitz formula the genus of the curve defined by (41) is seen to be that of $C_{c, \rho} / K$. Applying Liu02 10.3.44, there is a component in the stable reduction birationally given by (4). The stable reduction being a tree, the curve $C_{c, \rho} / K$ has good reduction over $K$.

2. The proof is divided into eight steps. Let $y$ be a root of $L_{c, \rho}(X)$.

Step I : One has $v(y)=v\left(a_{n} c\right) / q^{2}$.

By expanding $L_{c, \rho}(X)$, one shows that its Newton polygon has a single slope $v\left(a_{n} c\right) / q^{2}$. The polynomial $L_{c, \rho}(X)$ has degree $q^{2}$ and its leading (resp. constant) coefficient has valuation 0 (resp. $v\left(a_{n} c\right)$ ). One examines monomials from $a_{n} f_{c, \rho}^{q-1}(X)(c+X)$. Since $v(c)<v\left(\lambda^{p /(1+q)}\right)$, one checks that

$$
\forall 1 \leq i \leq q^{2}-1, \frac{v\left(a_{n}\right)}{q^{2}-i} \geq \frac{v\left(a_{n} c\right)}{q^{2}} .
$$

Then one examines monomials from $\left(\rho_{i} X\right)^{q / p^{i}} p^{d_{i}} f_{c, \rho}(X)^{q\left(p^{i}-1\right) / p^{i}}$. They have degree at least $q / p^{i}$, thus one checks that

$$
\forall 1 \leq i \leq n-1, \frac{q / p^{i} v\left(\rho_{i}\right)+d_{i} v(p)}{q^{2}-q / p^{i}} \geq \frac{v\left(a_{n} c\right)}{q^{2}} .
$$

The monomial $X^{q^{2}}$ in $s_{1, \rho}(X)^{q}$ corresponds to the point $(0,0)$ in the Newton polygon of $L_{c, \rho}(X)$, the other monomials of $s_{1, \rho}(X)^{q}$ produce a slope greater than $v\left(\rho_{i}\right) /\left(q-p^{i}\right)$ and one checks that

$$
\forall 0 \leq i \leq n-1, \frac{v\left(\rho_{i}\right)}{q-p^{i}} \geq \frac{v\left(a_{n} c\right)}{q^{2}} .
$$

Note that Step I implies that $v\left(f_{c, \rho}(y)\right)=0$, we will use this remark throughout this proof.

Step II : Define $S$ and $T$ by $\lambda^{p /(1+q)} T=(X-y)=S$. Then $f_{c, \rho}(S+y)$ is congruent modulo $\lambda^{p} \mathfrak{m}[T]$ to

$$
f_{c, \rho}(y)+s_{1, \rho}(y) S+\sum_{k=0}^{n-1} \rho_{k} S^{1+p^{k}}+\sum_{k=1}^{n-1} \rho_{k} y S^{p^{k}}+(c+y) S^{q}+S^{1+q} .
$$


Using the following formula for $A \in K^{\text {alg }}$ with $v(A)>0$ and $B \in\left(K^{\text {alg }}\right)^{\circ}[T]$

$$
k \geq 1,(A+B)^{p^{k}} \equiv\left(A^{p^{k-1}}+B^{p^{k-1}}\right)^{p} \bmod p^{2} \mathfrak{m}[T]
$$

one computes $\bmod \lambda^{p} \mathfrak{m}[T]$

$$
\begin{gathered}
f_{c, \rho}(y+S)=1+\sum_{k=0}^{n-1} \rho_{k}(y+S)^{1+p^{k}}+(y+S)^{1+q}+c(y+S)^{q} \\
\equiv 1+\rho_{0}(y+S)^{2}+\sum_{k=1}^{n-1} \rho_{k}(y+S)\left(y^{p^{k-1}}+S^{p^{k-1}}\right)^{p}+(y+S+c)\left(y^{q / p}+S^{q / p}\right)^{p} .
\end{gathered}
$$

Using Step I, one checks that for all $1 \leq k \leq n-1$

$$
\rho_{k}\left(y^{p^{k-1}}+S^{p^{k-1}}\right)^{p} \equiv \rho_{k}\left(y^{p^{k}}+S^{p^{k}}\right) \bmod \lambda^{p} \mathfrak{m}[T]
$$

and $\left(y^{q / p}+S^{q / p}\right)^{p} \equiv y^{q}+S^{q} \bmod \lambda^{p} \mathfrak{m}[T]$. It follows that

$f_{c, \rho}(y+S) \equiv 1+\rho_{0}(y+S)^{2}+\sum_{k=1}^{n-1} \rho_{k}(y+S)\left(y^{p^{k}}+S^{p^{k}}\right)+(y+c+S)\left(y^{q}+S^{q}\right)$.

One easily concludes from this last expression.

Step III : Let $R_{1}:=K[y]^{\circ}$. For all $0 \leq i \leq n$, one defines $A_{i}(S) \in R_{1}[S]$ and $B_{i} \in R_{1}$ by induction:

$$
\begin{gathered}
B_{n}:=-s_{1, \rho}(y), \quad \forall 1 \leq i \leq n-1, B_{i}:=\frac{f_{q, c}(y) B_{i+1}^{p}}{\left(-p f_{c, \rho}(y)\right)^{p}}-y \rho_{n-i}, \\
\quad \text { and } B_{0}:=\frac{f_{c, \rho}(y) B_{1}^{p}}{\left(-p f_{c, \rho}(y)\right)^{p}}, \\
A_{0}(S):=0 \text { and } \forall 0 \leq i \leq n-1 S A_{i+1}(S):=S A_{i}(S)-\frac{B_{i+1} S^{q / p^{i+1}}}{p f_{q, c}(y)^{(p-1) / p}} .
\end{gathered}
$$

Then for all $0 \leq i \leq n-1, v\left(B_{i+1}\right)=\left(1+\cdots+p^{i}\right) v(p) / p^{i}+v(c) / p^{i+1}$ and modulo $\lambda^{\frac{p q^{2}}{q+1}} \mathfrak{m}$ one has

$$
B_{n}^{q} \equiv \frac{a_{n}}{(-1)^{q}} f_{c, \rho}(y)^{q-1} B_{0}+\sum_{k=1}^{n-1}\left(\rho_{k} y\right)^{q / p^{k}}(-p)^{d_{k}} f_{c, \rho}(y)^{q\left(p^{k}-1\right) / p^{k}} .
$$

We prove the claim about $v\left(B_{i+1}\right)$ by induction on $i$. Using Step I, one checks that $\forall 0 \leq k \leq n-1, v\left(\rho_{k} y^{p^{k}}\right)>v\left(y^{q}\right)$, so $v\left(B_{n}\right)=v\left(y^{q}\right)$. Assume that 
we have shown the claim for $i$, then one checks that $v\left(\left(B_{i+1} / p\right)^{p}\right)<v\left(y \rho_{n-i}\right)$ and one deduces $v\left(B_{i}\right)$ from the definition of $B_{i}$. According to the expression of $v\left(B_{i}\right)$, one has $\forall 0 \leq i \leq n, A_{i}(S) \in R_{1}[S]$.

Then we prove the second part of Step III. From the definition of the $B_{i}$ 's one obtains that for all $1 \leq i \leq n-1$

$$
B_{n-i+1}^{q / p^{i-1}}=(-p)^{q / p^{i-1}} f_{c, \rho}(y)^{q(p-1) / p^{i}}\left(y \rho_{i}+B_{n-i}(y)\right)^{q / p^{i}} .
$$

Using Step I and $v\left(B_{n-1}\right)$ one checks that $\forall 1 \leq k \leq q / p-1$

$$
p^{q}\left(\begin{array}{c}
q / p \\
k
\end{array}\right)\left(y \rho_{1}\right)^{k} B_{n-1}^{q / p-k} \equiv 0 \bmod \lambda^{p q^{2} /(1+q)} \mathfrak{m},
$$

so $p^{q}\left(y \rho_{1}+B_{n-1}\right)^{q / p} \equiv p^{q}\left(\left(y \rho_{1}\right)^{q / p}+B_{n-1}^{q / p}\right) \bmod \lambda^{p q^{2} /(1+q)} \mathfrak{m}$. Thus, applying equation (6) with $i=1$, one gets

$$
\begin{aligned}
B_{n}^{q} & =(-p)^{q} f_{c, \rho}(y)^{q(p-1) / p}\left(y \rho_{1}+B_{n-1}\right)^{q / p} \\
& \equiv(-p)^{q} f_{c, \rho}(y)^{q(p-1) / p}\left(\left(y \rho_{1}\right)^{q / p}+B_{n-1}^{q / p}\right) \bmod \lambda^{p q^{2} /(1+q)} \mathfrak{m} .
\end{aligned}
$$

One checks using Step I and $v\left(B_{n-i}\right)$ that $\forall 1 \leq i \leq n-1$ and $1 \leq k \leq$ $q / p^{i}-1$

$$
p^{q+\cdots+q / p^{i-1}}\left(\begin{array}{c}
q / p^{i} \\
k
\end{array}\right) B_{n-i}^{q / p^{i}-k}\left(y \rho_{i}\right)^{k} \equiv 0 \bmod \lambda^{p q^{2} /(1+q)} \mathfrak{m}
$$

then by induction on $i$, using equation (ㅁ), one shows that modulo $\lambda^{p q^{2} /(1+q)} \mathfrak{m}$

$$
B_{n}^{q} \equiv(-p)^{p+\cdots+q} f_{c, \rho}(y)^{q-1} B_{0}+\sum_{k=1}^{n-1}\left(\rho_{k} y\right)^{q / p^{k}}(-p)^{d_{k}} f_{c, \rho}(y)^{q\left(p^{k}-1\right) / p^{k}} .
$$

Step IV : One has modulo $\lambda^{p} \mathfrak{m}[T]$

$$
f_{c, \rho}(S+y) \equiv f_{c, \rho}(y)+s_{1, \rho}(y) S+\sum_{k=0}^{n-1} \rho_{k} S^{1+p^{k}}+\sum_{k=1}^{n-1} y \rho_{k} S^{p^{k}}+B_{0} S^{q}+S^{1+q} .
$$

Since $L_{c, \rho}(y)=0$, one has

$$
s_{1, \rho}(y)^{q}=a_{n} f_{c, \rho}(y)^{q-1}(c+y)+(-1)^{q} \sum_{k=1}^{n-1}\left(\rho_{k} y\right)^{q / p^{k}}(-p)^{d_{k}} f_{c, \rho}(y)^{q\left(p^{k}-1\right) / p^{k}} .
$$

Using $B_{n}:=-s_{1, \rho}(y)$, equations (5) and (17) one gets

$$
a_{n} f_{c, \rho}(y)^{q-1}\left(c+y-B_{0}\right) \equiv 0 \bmod \lambda^{p q^{2} /(q+1)} \mathfrak{m} .
$$


which is equivalent to $S^{q}\left(y+c-B_{0}\right) \equiv 0 \bmod \lambda^{p} \mathfrak{m}[T]$. Then, Step IV follows from Step II.

Step V : One has

$$
f_{c, \rho}(S+y) \equiv\left(f_{c, \rho}(y)^{1 / p}+S A_{n}(S)\right)^{p}+\sum_{k=0}^{n-1} \rho_{k} S^{1+p^{k}}+S^{1+q} \bmod \lambda^{p} \mathfrak{m}[T] .
$$

Let $R:=\sum_{k=0}^{n-1} \rho_{k} S^{1+p^{k}}+S^{1+q}+s_{1, \rho}(y) S$. Since $B_{n}=-s_{1, \rho}(y)$ one has

$$
\begin{aligned}
& \left(f_{c, \rho}(y)^{1 / p}+S A_{n}(S)\right)^{p}+\sum_{k=0}^{n-1} \rho_{k} S^{1+p^{k}}+S^{1+q} \\
= & \left(f_{c, \rho}(y)^{1 / p}+S A_{n}(S)\right)^{p}+B_{n} S+R \\
= & \left(f_{c, \rho}(y)^{1 / p}+S A_{n-1}(S)-\frac{B_{n} S}{p f_{q, c}(y)^{(p-1) / p}}\right)^{p}+B_{n} S+R \\
= & \left(f_{c, \rho}(y)^{1 / p}+S A_{n-1}(S)\right)^{p}+\left(\frac{-B_{n} S}{p f_{q, c}(y)^{(p-1) / p}}\right)^{p}+B_{n} S+R+\Sigma,
\end{aligned}
$$

where

$$
\Sigma=\sum_{k=1}^{p-1}\left(\begin{array}{l}
p \\
k
\end{array}\right)\left(f_{c, \rho}(y)^{1 / p}+S A_{n-1}(S)\right)^{p-k}\left(\frac{-B_{n} S}{p f_{q, c}(y)^{(p-1) / p}}\right)^{k} .
$$

Using the expression of $v\left(B_{n}\right)$ computed in Step III, one checks that the terms with $k \geq 2$ in (9) are zero modulo $\lambda^{p} \mathfrak{m}[T]$. It implies the following relations

$$
\begin{aligned}
\Sigma+B_{n} S & \equiv B_{n} S\left[1-\frac{\left(f_{c, \rho}(y)^{1 / p}+S A_{n-1}(S)\right)^{p-1}}{f_{c, \rho}(y)^{(p-1) / p}}\right] \\
& \equiv \frac{B_{n} S}{f_{c, \rho}(y)^{(p-1) / p}}\left[f_{c, \rho}(y)^{(p-1) / p}-\left(f_{c, \rho}(y)^{1 / p}+S A_{n-1}(S)\right)^{p-1}\right] \\
& \equiv \frac{B_{n} S}{f_{c, \rho}(y)^{(p-1) / p}}\left[-\sum_{k=1}^{p-1}\left(\begin{array}{c}
p-1 \\
k
\end{array}\right) f_{c, \rho}(y)^{(p-1-k) / p}\left(S A_{n-1}(S)^{k}\right]\right. \\
& \equiv 0 \bmod \lambda^{p} \mathfrak{m}[T], \text { since for } k \geq 1, B_{n} S^{k+1} \equiv 0 \bmod \lambda^{p} \mathfrak{m}[T] .
\end{aligned}
$$

According to the definition of $B_{n-1}$ (see Step III) one obtains

$$
(8) \equiv\left(f_{c, \rho}(y)^{1 / p}+S A_{n-1}(S)\right)^{p}+R+B_{n-1} S^{p}+y \rho_{1} S^{p} \bmod \lambda^{p} \mathfrak{m}[T] .
$$


Using the same process, one shows by induction on $i$ that (8) is congruent to

$$
\left(f_{c, \rho}(y)^{1 / p}+S A_{i+1}(S)\right)^{p}+B_{i+1} S^{p^{n-i-1}}+\sum_{k=1}^{n-i-1} y \rho_{k} S^{p^{k}}+R \bmod \lambda^{p} \mathfrak{m}[T] .
$$

Thus, one applies equation (11) with $i=0$

$$
\text { (8) } \equiv\left(f_{c, \rho}(y)^{1 / p}+S A_{1}(S)\right)^{p}+B_{1} S^{q / p}+\sum_{k=1}^{n-1} y \rho_{k} S^{p^{k}}+R \bmod \lambda^{p} \mathfrak{m}[T] .
$$

One defines $\Sigma^{\prime}$ by $\left(f_{c, \rho}(y)^{1 / p}+S A_{1}(S)\right)^{p}=f_{c, \rho}(y)+\left(S A_{1}(S)\right)^{p}+\Sigma^{\prime}$. From $p f_{c, \rho}(y)^{(p-1) / p} S A_{1}(S)=-B_{1} S^{q / p}$ (see the definition of $S A_{1}(S)$ ) one gets

$$
\Sigma^{\prime}+B_{1} S^{q / p}=\sum_{k=2}^{p-1}\left(\begin{array}{l}
p \\
k
\end{array}\right) f_{c, \rho}(y)^{(p-k) / p}\left(S A_{1}(S)\right)^{k},
$$

so using the expression of $v\left(B_{1}\right)$ computed in Step III, one checks that $\Sigma^{\prime}+B_{1} S^{q / p} \equiv 0 \bmod \lambda^{p} \mathfrak{m}[T]$. From the definition of $S A_{1}(S)$ and $B_{0}$ one has $\left(S A_{1}(S)\right)^{p}=B_{0} S^{q}$, thus

$$
\text { (요 } \equiv f_{c, \rho}(y)+B_{0} S^{q}+\sum_{k=1}^{n-1} y \rho_{k} S^{p^{k}}+R \bmod \lambda^{p} \mathfrak{m}[T] \text {. }
$$

Then, Step V follows from Step IV and this last relation.

Step VI : The curve $C_{c, \rho} / K$ has good reduction over $K\left(y, f_{c, \rho}(y)^{1 / p}\right)$. According to Step $\mathbf{V}$, the change of variables in $K\left(y, f_{c, \rho}(y)^{1 / p}\right)$

$$
X=\lambda^{p /(1+q)} T+y=S+y \text { and } Y=\lambda W+f_{c, \rho}(y)^{1 / p}+S A_{n}(S),
$$

induces in reduction $w^{p}-w=\sum_{k=0}^{n-1} \bar{u}_{k} t^{1+p^{k}}+t^{1+q}$ with genus $g\left(C_{c, \rho}\right)$. So [Liu02 10.3.44 implies that this change of variables gives the stable model. Note that the $\rho_{k}$ 's were chosen to obtain this equation for the special fiber of the stable model.

Step VII : For any distinct roots $y_{i}, y_{j}$ of $L_{c, \rho}(X), v\left(y_{i}-y_{j}\right)=v\left(\lambda^{p /(1+q)}\right)$. The changes of variables $\lambda^{p /(1+q)} T=X-y_{i}$ and $\lambda^{p /(1+q)} T=X-y_{j}$ induce equivalent Gauss valuations of $K\left(C_{c, \rho}\right)$, else applying [Liu02] 10.3.44 would contradict the uniqueness of the stable model. Thus $v\left(y_{i}-y_{j}\right) \geq v\left(\lambda^{p /(1+q)}\right)$.

One checks that $v\left(f_{c, \rho}^{\prime}(y)\right)>0, \forall 0 \leq k \leq n-1 v\left(\rho_{k}^{q / p^{k}} p^{d_{k}} q / p^{k}\right)>v\left(a_{n}\right)$, $v\left(s_{1, \rho}^{\prime}(y)\right)>0, v\left(s_{1, \rho}(y)\right)=v\left(y^{q}\right)$ and $v\left(q s_{1, \rho}(y)^{q-1} s_{1, \rho}^{\prime}(y)\right)>v\left(a_{n}\right)$, so

$$
v\left(L_{c, \rho}^{\prime}(y)\right)=v\left(a_{n}\right)=\left(q^{2}-1\right) v\left(\lambda^{p /(1+q)}\right) .
$$


Taking into account that $L_{c, \rho}^{\prime}\left(y_{i}\right)=\prod_{j \neq i}\left(y_{i}-y_{j}\right)$ and $\operatorname{deg} L_{c, \rho}(X)=q^{2}$, one obtains $v\left(y_{i}-y_{j}\right)=v\left(\lambda^{p /(1+q)}\right)$.

Step VIII : If $L_{c, \rho}(X)$ is irreducible over $K$, then $K\left(y, f_{c, \rho}(y)^{1 / p}\right)$ is the monodromy extension $M$ of $C_{c, \rho} / K$ and $G:=\operatorname{Gal}(M / K)$ is an extra-special p-group of order $p q^{2}$.

Let $\left(y_{i}\right)_{i=1, \ldots, q^{2}}$ be the roots of $L_{c, \rho}(X), L:=K\left(y_{1}, \ldots, y_{q^{2}}\right)$ and $M / K$ be the monodromy extension of $C_{c, \rho} / K$. Any $\tau \in \operatorname{Gal}(L / K)-\{1\}$ is such that $\tau\left(y_{i}\right)=y_{j}$ for some $i \neq j$. Thus, the change of variables

$$
X=\lambda^{p /(1+q)} T+y_{i} \text { and } Y=\lambda W+f_{c, \rho}(y)^{1 / p}+S A_{n}(S),
$$

induces the stable model and $\tau$ acts on it by :

$$
\tau(T)=\frac{X-y_{j}}{\lambda^{p /(1+q)}}, \quad \text { hence } \quad T-\tau(T)=\frac{y_{j}-y_{i}}{\lambda^{p /(1+q)}} .
$$

According to Step VII, $\tau$ acts non-trivially on the stable reduction. It follows that $L \subseteq M$. Indeed if $\operatorname{Gal}\left(K^{\text {alg }} / M\right) \nsubseteq \mathrm{Gal}\left(K^{\text {alg }} / L\right)$ it would exist $\sigma \in \operatorname{Gal}\left(K^{\text {alg }} / M\right)$ inducing $\bar{\sigma} \neq \operatorname{Id} \in \operatorname{Gal}(L / K)$, which would contradict the characterization of $\operatorname{Gal}\left(K^{\text {alg }} / M\right)$ (see remark after Theorem 2.1) .

According to [LM05], the $p$-Sylow subgroup $\operatorname{Aut}_{k}\left(\mathcal{C}_{k}\right)_{1}^{\#}$ of $\operatorname{Aut}_{k}\left(\mathcal{C}_{k}\right)^{\#}$ is an extra-special $p$-group of order $p q^{2}$. Moreover, one has :

$$
0 \rightarrow \mathrm{Z}\left(\operatorname{Aut}_{k}\left(\mathcal{C}_{k}\right)_{1}^{\#}\right) \rightarrow \operatorname{Aut}_{k}\left(\mathcal{C}_{k}\right)_{1}^{\#} \rightarrow(\mathbb{Z} / p \mathbb{Z})^{2 n} \rightarrow 0,
$$

where $(\mathbb{Z} / p \mathbb{Z})^{2 n}$ is identified with the group of translations $t \mapsto t+a$ extending to elements of $\operatorname{Aut}_{k}\left(\mathcal{C}_{k}\right)_{1}^{\#}$. Therefore we have morphisms

$$
\operatorname{Gal}(M / K) \stackrel{i}{\rightarrow} \operatorname{Aut}_{k}\left(\mathcal{C}_{k}\right)_{1}^{\#} \stackrel{\varphi}{\rightarrow} \operatorname{Aut}_{k}\left(\mathcal{C}_{k}\right)_{1}^{\#} / \mathrm{Z}\left(\operatorname{Aut}_{k}\left(\mathcal{C}_{k}\right)_{1}^{\#}\right)
$$

The composition is seen to be surjective since the image contains the $q^{2}$ translations $t \mapsto t+\overline{\left(y_{i}-y_{1}\right) / \lambda^{p /(1+q)}}$. Consequently, $i(\operatorname{Gal}(M / K))$ is a subgroup of $\operatorname{Aut}_{k}\left(\mathcal{C}_{k}\right)_{1}^{\#}$ of index at most $p$. So it contains $\Phi\left(\operatorname{Aut}_{k}\left(\mathcal{C}_{k}\right)_{1}^{\#}\right)=$ $\mathrm{Z}\left(\operatorname{Aut}_{k}\left(\mathcal{C}_{k}\right)_{1}^{\#}\right)=\operatorname{Ker} \varphi$. It implies that $i$ is an isomorphism and $[M: K]=p q^{2}$. By Step VI, one has $M \subseteq K\left(y, f_{q, c}(y)^{1 / p}\right)$, hence $M=K\left(y, f_{q, c}(y)^{1 / p}\right)$.

We show that $K\left(y_{1}\right) / K$ is Galois and that $\operatorname{Gal}\left(M / K\left(y_{1}\right)\right)=\mathrm{Z}(G)$. Indeed, $M / K\left(y_{1}\right)$ is $p$-cyclic and generated by $\sigma$ defined by :

$$
\sigma\left(y_{1}\right)=y_{1} \text { and } \sigma\left(f_{c, \rho}\left(y_{1}\right)^{1 / p}\right)=\zeta_{p} f_{c, \rho}\left(y_{1}\right)^{1 / p} .
$$

According to Step VI, $\sigma$ acts on the stable model by :

$$
\sigma(S)=S, \quad \sigma(Y)=Y=\lambda \sigma(W)+\zeta_{p} f_{c, \rho}\left(y_{1}\right)^{1 / p}+S A_{n}(S) .
$$


Hence

$$
\sigma(W)=W-f_{c, \rho}\left(y_{1}\right)^{1 / p} .
$$

It follows that, in reduction, $\sigma$ induces a morphism that generates $\mathrm{Z}\left(\operatorname{Aut}_{k}\left(\mathcal{C}_{k}\right)_{1}^{\#}\right)$. It implies that $K\left(y_{1}\right) / K$ is Galois, $\operatorname{Gal}\left(M / K\left(y_{1}\right)\right)=\mathrm{Z}(G)$ and $\operatorname{Gal}\left(K\left(y_{1}\right) / K\right) \simeq$ $(\mathbb{Z} / p \mathbb{Z})^{2 n}$.

3. Let $L_{\rho}(X):=L_{1, \rho}(X), f_{\rho}(X):=f_{1, \rho}(X), s_{\rho}(y):=s_{1, \rho}(y), y$ be a root of $L_{\rho}(X)$ and $b_{n}:=(-1)(-p)^{1+p+\cdots+p^{n-1}}$. Note that $b_{n}^{p}=a_{n}, L=K(y)$ and we do not assume $p=2$ until Step E.

Step A : The polynomial $L_{\rho}(X)$ is irreducible over $K$.

Let $\tilde{s}:=s_{\rho}(y)-y^{q}, \sigma:=\sum_{k=1}^{q}\left(\begin{array}{l}q \\ k\end{array}\right) \tilde{s}^{k} y^{q(q-k)}$ and $R_{1}:=\sum_{k=1}^{p-1}\left(\begin{array}{l}p \\ k\end{array}\right) y^{k q^{2} / p}\left(-b_{n}\right)^{p-k}$. Since $L_{\rho}(y)=0$ one has

$$
y^{q^{2}}+\sigma=s_{\rho}(y)^{q}=a_{n} f_{\rho}(y)(1+y)+\sum_{k=1}^{n-1}\left(\rho_{k} y\right)^{q / p^{k}}(-p)^{d_{k}}(-1)^{q} f_{\rho}(y)^{q\left(p^{k}-1\right) / p^{k}} .
$$

It implies that $\left(y^{q^{2} / p}-b_{n}\right)^{p}$ equals

$$
a_{n}\left[f_{\rho}(y)(1+y)+(-1)^{p}\right]+\sum_{k=1}^{n-1}\left(\rho_{k} y\right)^{q / p^{k}}(-p)^{d_{k}}(-1)^{q} f_{\rho}(y)^{q\left(p^{k}-1\right) / p^{k}}+R_{1}-\sigma .
$$

We are going to remove monomials with valuation greater than $v\left(a_{n} y\right)$ in the above expression by taking $p$-th roots. Note that if $\forall i \geq 1, \rho_{i}=0$, then one could skip most of Step A (see equation (14)). Assume that $\rho_{i} \neq 0$ for some $i \geq 1$, let $j:=\max \left\{1 \leq i \leq n-1, \rho_{i} \neq 0\right\}$ and $l:=\min \left\{1 \leq i \leq n-1, \rho_{i} \neq 0\right\}$. The following relations are straight forward computations using Step I :

$$
\begin{aligned}
& v\left(f_{\rho}(y)(1+y)+(-1)^{p}\right)=v(y), \quad v(\tilde{s})=v\left(\rho_{j} y^{p^{j}}\right), \quad v(\sigma)=q v(\tilde{s}), \\
& v\left(\sum_{k=1}^{n-1}\left(\rho_{k} y\right)^{q / p^{k}}(-p)^{d_{k}}(-1)^{q} f_{\rho}(y)^{q\left(p^{k}-1\right) / p^{k}}\right)=v\left(\left(\rho_{l} y\right)^{p^{n-l}} p^{d_{l}}\right) .
\end{aligned}
$$

Then one checks that

$$
v\left(R_{1}\right)>v\left(a_{n} y\right)>v\left(\left(\rho_{l} y\right)^{p^{n-l}} p^{d_{l}}\right)>v(\sigma) .
$$

It implies that $v\left(\left(y^{q^{2} / p}-b_{n}\right)^{p}\right)=q v(\tilde{s})$, so one considers $\left(y^{q^{2} / p}-b_{n}+\tilde{s}^{q / p}\right)^{p}$. By expanding this last expression, using (12), (13) and taking into account

$$
v\left(\sum_{k=1}^{q-1}\left(\begin{array}{l}
q \\
k
\end{array}\right) \tilde{s}^{k} y^{q(q-k)}\right)>v\left(a_{n} y\right), v\left(\sum_{k=1}^{p}\left(\begin{array}{l}
p \\
k
\end{array}\right)\left(y^{q^{2} / p}-b_{n}\right)^{k} \tilde{s}^{(p-k) q / p}\right)>v\left(a_{n} y\right),
$$


one obtains that $p v\left(y^{q^{2} / p}-b_{n}+\tilde{s}^{q / p}\right)=v\left(\left(\rho_{l} y\right)^{p^{n-l}} p^{d_{l}}\right)$, leading us to consider

$$
\left(y^{q^{2} / p}-b_{n}+\tilde{s}^{q / p}+\left(\rho_{l} y\right)^{q / p^{l+1}}(-p)^{d_{l} / p} f_{\rho}(y)^{q\left(p^{l}-1\right) / p^{l+1}}\right)^{p} .
$$

By expanding this expression and using (12) and (13) one easily checks that it has valuation $v\left(\left(\rho_{l_{1}} y\right)^{p^{n-l_{1}}} p^{d_{l_{1}}}\right)$ where $l_{1}:=\min \left\{l+1 \leq i \leq n-1, \rho_{i} \neq 0\right\}$. By induction one shows that

$$
t:=y^{q^{2} / p}-b_{n}+\tilde{s}^{q / p}+\sum_{k=1}^{n-1}\left(\rho_{k} y\right)^{q / p^{k+1}}(-p)^{d_{k} / p} f_{\rho}(y)^{q\left(p^{k}-1\right) / p^{k+1}}
$$

satisfies $p v(t)=v\left(a_{n} y\right)$. Then $v_{L}\left(p^{q^{2}} t^{-(p-1)(q+1)}\right)=v_{L}(p) / q^{2}=\left[L: \mathbb{Q}_{p}^{\text {ur }}\right] / q^{2}$, so $q^{2}$ divides $[L: K]$. It implies that $L_{\rho}(X)$ is irreducible over $K$.

Step B : Reduction step.

The last non-trivial group $G_{i_{0}}$ of the lower ramification filtration $\left(G_{i}\right)_{i \geq 0}$ of $G:=\operatorname{Gal}(M / K)$ is a subgroup of $\mathrm{Z}(G)$ (Ser79] IV $\S 2$ Corollary 2 of Proposition 9$)$ and as $\mathrm{Z}(G) \simeq \mathbb{Z} / p \mathbb{Z}$, it follows that $G_{i_{0}}=\mathrm{Z}(G)$.

According to Step VIII the group $H:=\operatorname{Gal}(M / L)$ is $\mathrm{Z}(G)$. Consequently, the filtration $\left(G_{i}\right)_{i \geq 0}$ can be deduced from that of $M / L$ and $L / K$ (see [Ser79] IV §2 Proposition 2 and Corollary of Proposition 3).

Step C : Let $\sigma \in \operatorname{Gal}(L / K)-\{1\}$, then $v(\sigma(t)-t)=q^{2} v\left(\pi_{K}\right)$.

Let $y^{\prime}:=\sigma(y)$, one deduces the following easy lemma from Step VII.

Lemma 3.1. For any $n \geq 0, v\left(y^{n}-y^{\prime n}\right) \geq n v(y)$.

Recall the definition $\tilde{s}:=2 \rho_{0} y+\sum_{k=1}^{n-1} \rho_{k} y^{p^{k}}$. First one shows that modulo $\left(y-y^{\prime}\right)^{q^{2} / p} \mathfrak{m}$ one has

$$
\sigma(\tilde{s})^{q / p}-\tilde{s}^{q / p} \equiv\left(2 \rho_{0}\right)^{q / p}\left(y^{\prime q / p}-y^{q / p}\right)+\sum_{k=1}^{n-1} \rho_{k}^{q / p}\left(y^{\prime q p^{k} / p}-y^{q p^{k} / p}\right) .
$$

Indeed, let $\left(m_{i}\right)_{i=0, \ldots, n-1} \in \mathbb{N}^{n}$ such that $m_{0}+m_{1}+\cdots+m_{n-1}=q / p$ and $t:=m_{0}+m_{1} p+\cdots+m_{n-1} p^{n-1}$, then using lemma 3.1 one checks that

$$
v\left(p \rho_{0}^{m_{0}} \rho_{1}^{m_{1}} \ldots \rho_{n-1}^{m_{n-1}}\left(y^{t}-y^{\prime t}\right)\right)>\frac{q^{2}}{p} v\left(y-y^{\prime}\right) .
$$

This inequality implies (15).

Let $1 \leq k \leq n-1$ and write $f_{\rho}(y)^{\left(p^{k}-1\right) q / p^{k+1}}=1+\sum_{i \in I_{k}} \alpha_{i, k} y^{i}$, for some 
set $I_{k}$. Then

$$
\begin{aligned}
& y^{q / p^{k+1}} f_{\rho}\left(y^{\prime}\right)^{\left(p^{k}-1\right) q / p^{k+1}}-y^{q / p^{k+1}} f_{\rho}(y)^{\left(p^{k}-1\right) q / p^{k+1}} \\
= & y^{\prime q / p^{k+1}}-y^{q / p^{k+1}}+\sum_{i \in I_{k}} \alpha_{i, k}\left(y^{i}-y^{i}\right) .
\end{aligned}
$$

Let $i \in I_{k}$. Consider the case when $v\left(\alpha_{i, k}\right) \geq v\left(\rho_{h}\right)$ for some $0 \leq h \leq$ $n-1$, then using Step VII, one checks that $\forall 1 \leq k \leq n-1, v\left(\alpha_{i, k}\right) \geq$ $v\left(\rho_{h}\right)>q v\left(y^{\prime}-y\right) / p^{k+1}$. If this case does not occur, then according to the expression of $f_{\rho}(y)$ one has $i \geq q / p^{k+1}+q$ and using lemma 3.1 one checks that $v\left(y^{\prime i}-y^{i}\right)>q v\left(y^{\prime}-y\right) / p^{k+1}$. In any case $v\left(\alpha_{i, k}\left(y^{\prime i}-y^{i}\right)\right)>q v\left(y^{\prime}-y\right) / p^{k+1}$ and one checks that

$$
v\left(p^{d_{k} / p} \rho_{k}^{q / p^{k+1}} \alpha_{i, k}\left(y^{i}-y^{i}\right)\right)>q^{2} v\left(y^{\prime}-y\right) / p .
$$

Taking into account (14), (15) and (16), one gets $\bmod \left(y^{\prime}-y\right)^{q^{2} / p_{\mathfrak{m}}}$

$$
\begin{aligned}
\sigma(t)- & t \equiv y^{\prime q^{2} / p}-y^{q^{2} / p}+\left(2 \rho_{0}\right)^{q / p}\left(y^{\prime q / p}-y^{q / p}\right) \\
& +\sum_{k=1}^{n-1} \rho_{k}^{q / p}\left(y^{\prime q p^{k} / p}-y^{q p^{k} / p}\right)+\sum_{k=1}^{n-1}(-p)^{d_{k} / p} \rho_{k}^{q / p^{k+1}}\left(y^{\prime q / p^{k+1}}-y^{q / p^{k+1}}\right) .
\end{aligned}
$$

Using lemma 3.1, it is now straight forward to check the following relations $\bmod \left(y^{\prime}-y\right)^{q^{2} / p} \mathfrak{m}$.

$$
\begin{aligned}
y^{\prime q^{2} / p}-y^{q^{2} / p} & \equiv\left(y^{\prime}-y\right)^{q^{2} / p}, \\
\rho_{k}^{q / p}\left(y^{\prime q p^{k} / p}-y^{q p^{k} / p}\right) & \equiv \rho_{k}^{q / p}\left(y^{\prime}-y\right)^{q p^{k} / p}, \\
(-p)^{d_{k} / p} \rho_{k}^{q / p^{k+1}}\left(y^{q / p^{k+1}}-y^{q / p^{k+1}}\right) & \equiv(-p)^{d_{k} / p} \rho_{k}^{q / p^{k+1}}\left(y^{\prime}-y\right)^{q / p^{k+1}} .
\end{aligned}
$$

Using Step VII, one sees that each of these three elements has valuation $q^{2} v\left(y^{\prime}-y\right) / p$, thus one gets

$$
\begin{aligned}
(\sigma(t)-t)^{p} & \equiv\left(y^{\prime}-y\right)^{q^{2}}+\left(2 \rho_{0}\right)^{q}\left(y^{\prime}-y\right)^{q}+\sum_{k=1}^{n-1} \rho_{k}^{q}\left(y^{\prime}-y\right)^{q p^{k}} \\
& +\sum_{k=1}^{n-1}(-p)^{d_{k}} \rho_{k}^{q / p^{k}}\left(y^{\prime}-y\right)^{q / p^{k}} \bmod \left(y^{\prime}-y\right)^{q^{2}} \mathfrak{m} .
\end{aligned}
$$

Now recall Step VII, the definitions of the $\rho_{k}$ 's and of $\lambda$, then for some $v \in R^{\times}$and $\Sigma \in R$

$$
\rho_{k}=u_{k} \lambda^{p\left(q-p^{k}\right) /(1+q)}, \quad y^{\prime}-y=v \lambda^{p /(1+q)} \text { and }-p=\lambda^{p-1}+p \lambda \Sigma .
$$


Since $q^{2} v\left(y^{\prime}-y\right)=\frac{p q^{2}}{1+q} v(\lambda)$, equation (18) becomes

$$
(\sigma(t)-t)^{p} \equiv \lambda^{\frac{q^{2} p}{1+q}}\left[v^{q^{2}}+\left(2 u_{0} v\right)^{q}+\sum_{k=1}^{n-1}\left(u_{k}^{q} v^{q p^{k}}+\left(u_{k} v\right)^{q / p^{k}}\right)\right] \bmod \lambda^{\frac{q^{2} p}{1+q}} \mathfrak{m} .
$$

From the action of $\sigma$ on the stable reduction (see Step VIII), one has that the automorphism of $\mathbb{P}_{k}^{1}$ given by $t \mapsto t+\bar{v}$ has a prolongation to $A_{u} / k$, so Proposition 2.4 implies that

$$
\bar{v}^{q^{2}}+\left(2 \bar{u}_{0} \bar{v}\right)^{q}+\sum_{k=1}^{n-1}\left(\bar{u}_{k}^{q} \bar{v}^{q p^{k}}+\left(\bar{u}_{k} \bar{v}\right)^{q / p^{k}}\right)+\bar{v}=0 .
$$

Assume that $\bar{v}^{q^{2}}+\left(2 \bar{u}_{0} \bar{v}\right)^{q}+\sum_{k=1}^{n-1}\left(\bar{u}_{k}^{q} \bar{v}^{q p^{k}}+\left(\bar{u}_{k} \bar{v}\right)^{q / p^{k}}\right)=0$, then from (19) one has $\bar{v}=0$, which contradicts $v \in R^{\times}$. It implies that $v(\sigma(t)-t)=$ $q^{2} v(\lambda) /(1+q)=q^{2} v\left(y-y^{\prime}\right) / p=q^{2} v\left(\pi_{K}\right)$.

Step D : The ramification filtration of $L / K$ is :

$$
(G / H)_{0}=(G / H)_{1} \supsetneq(G / H)_{2}=\{1\} .
$$

Since $K / \mathbb{Q}_{p}^{\text {ur }}$ is tamely ramified of degree $(p-1)(q+1)$, one has $K=\mathbb{Q}_{p}^{\text {ur }}\left(\pi_{K}\right)$ with $\pi_{K}^{(p-1)(q+1)}=p$ for some uniformizer $\pi_{K}$ of $K$. In particular $z:=\pi_{K}^{q^{2}} / t$, is a uniformizer of $L$. Let $\sigma \in \operatorname{Gal}(L / K)-\{1\}$, then

$$
\sigma(z)-z=\frac{t-\sigma(t)}{\sigma(t) t} \pi_{K}^{q^{2}}=\frac{t-\sigma(t)}{\pi_{K}^{q^{2}}} \frac{\pi_{K}^{q^{2}}}{t} \frac{\pi_{K}^{q^{2}}}{\sigma(t)} .
$$

Using Step C one obtains $v(\sigma(z)-z)=2 v(z)$, i.e. $(G / H)_{2}=\{1\}$.

Step E : From now on, we assume $p=2$. Let $s:=(q+1)\left(2 q^{2}-1\right)$. There exist $u, h \in L$ and $r \in \pi_{L}^{s} \mathfrak{m}$ such that $v_{L}\left(2 y^{q / 2} h\right)=s$ and

$$
f_{\rho}(y) u^{2}=1+\rho_{n-1} y^{1+q / 2}+2 y^{q / 2} h+r .
$$

To prove the first statement we note that, from the definition of $f_{\rho}(y)$, one has $f_{\rho}(y)=1+T$ with $v(T)=q v(y)$ and $L_{\rho}(y)=0$, thus

$$
\begin{aligned}
& \qquad\left(\frac{s_{\rho}^{q / 2}(y)}{b_{n}}\right)^{2}=f_{\rho}(y)^{q-1}(1+y)+\sum_{k=1}^{n-1} \frac{\left(\rho_{k} y\right)^{q / 2^{k}}}{2^{2+\cdots+2^{n-k}}} f_{\rho}(y)^{q\left(2^{k}-1\right) / 2^{k}}, \\
& \text { and } f_{\rho}(y)^{q-1}(1+y)=1+y+\sum_{k=1}^{q-1}\left(\begin{array}{c}
q-1 \\
k
\end{array}\right) T^{k}(1+y) .
\end{aligned}
$$


Then, we put $\tilde{\Sigma}:=\sum_{k=1}^{q-1}\left(\begin{array}{c}q-1 \\ k\end{array}\right) T^{k}(1+y)$ and

$$
h:=\frac{s_{\rho}^{q / 2}(y)}{b_{n}}+\sum_{k=1}^{n-1} \frac{\left(\rho_{k} y\right)^{q / 2^{k+1}}}{2^{1+\cdots+2^{n-k-1}}} f_{\rho}(y)^{q\left(2^{k}-1\right) / 2^{k+1}}-1 .
$$

Then one computes

$$
\begin{aligned}
h^{2} & =\left[\frac{s_{\rho}^{q / 2}(y)}{b_{n}}+\sum_{k=1}^{n-1} \frac{\left(\rho_{k} y\right)^{q / 2^{k+1}}}{2^{1+\cdots+2^{n-k-1}}} f_{\rho}(y)^{q\left(2^{k}-1\right) / 2^{k+1}}\right]^{2}+1-2(h+1) \\
& =\left(\frac{s_{\rho}^{q / 2}(y)}{b_{n}}\right)^{2}+\sum_{k=1}^{n-1} \frac{\left(\rho_{k} y\right)^{q / 2^{k}}}{2^{2+\cdots+2^{n-k}}} f_{\rho}(y)^{q\left(2^{k}-1\right) / 2^{k}}+\Sigma_{1}+1-2(h+1) \\
& =2+y+2 \sum_{k=1}^{n-1} \frac{\left(\rho_{k} y\right)^{q / 2^{k}}}{2^{2+\cdots+2^{n-k}}} f_{\rho}(y)^{q\left(2^{k}-1\right) / 2^{k}}+\Sigma_{1}+\tilde{\Sigma}-2(h+1) .
\end{aligned}
$$

In Step III, we proved that $v\left(B_{n}\right)=q v(y)=2 v\left(b_{n}\right) / q$ where $B_{n}=-s_{\rho}(y)$, so $v\left(\frac{s_{\rho}^{q / 2}(y)}{b_{n}}\right)=0$ and one checks using Step I that

$$
v(2)>v(y), \text { and } \forall 1 \leq k \leq n-1, v\left(\frac{\left(\rho_{k} y\right)^{q / 2^{k+1}}}{2^{1+\cdots+2^{n-k-1}}}\right) \geq 0,
$$

thus $v(h+1) \geq 0$ and $v(2(h+1)) \geq v(2)>v(y)$. One checks in the same way that $v\left(\Sigma_{1}\right)>v(y)$. One has $v(\tilde{\Sigma}) \geq v(T)>v(y)$, so $v\left(h^{2}\right)=v(y)$ and $v_{L}\left(2 y^{q / 2} h\right)=s$.

To prove the second statement of Step $\mathbf{E}$, we first remark that $\forall i \geq 1$ $f_{\rho}(y)^{i}=1+\sum_{k=1}^{i}\left(\begin{array}{l}i \\ k\end{array}\right) T^{k}=1+\Sigma_{i}$, whence $v\left(\sum_{i}\right) \geq v(T)$. Since, for all $0 \leq k \leq n-1, v\left(\rho_{k} y^{p^{k}}\right)>q v(y)$ one has $\bmod \pi_{L}^{s} \mathfrak{m}$

$$
\frac{s_{\rho}^{q / 2}(y)}{b_{n}} 2 y^{q / 2} \equiv\left[\left(2 \rho_{0} y\right)^{q / 2}+\sum_{k=1}^{n-1}\left(\rho_{k} y^{2^{k}}\right)^{q / 2}+y^{q^{2} / 2}\right] \frac{y^{q / 2}}{2^{2+\cdots+2^{n-1}}} .
$$

One also checks that $\forall i \geq 1, v_{L}\left(2 y^{q / 2} \Sigma_{i}\right)>s$, then according to (20), $\forall i \geq 1$ and $1 \leq k \leq n-1$

$$
v_{L}\left(\frac{\left(\rho_{k} y\right)^{q / 2^{k+1}}}{2^{1+\cdots+2^{n-k-1}}} 2 y^{q / 2} \Sigma_{i}\right)>s \text { and one checks that } v_{L}\left(\frac{\left(2 \rho_{0}\right)^{q / 2} y^{q}}{2^{2+\cdots+2^{n-1}}}\right)>s .
$$

Thus, applying relations (21), (22) and the definition of $h$, one has

$$
\begin{aligned}
2 h y^{q / 2} \equiv & {\left[\sum_{k=1}^{n-1}\left(\rho_{k} y^{2^{k}}\right)^{q / 2}+y^{q^{2} / 2}\right] \frac{y^{q / 2}}{2^{2+\cdots+2^{n-1}}} } \\
& +\sum_{k=1}^{n-1} \frac{\left(\rho_{k} y\right)^{q / 2^{k+1}}}{2^{1+\cdots+2^{n-k-1}}} 2 y^{q / 2}-2 y^{q / 2} \bmod \pi_{L}^{s} \mathfrak{m} .
\end{aligned}
$$


Finally one puts

$$
u:=1-y^{q / 2}-\sum_{k=0}^{n-2} \frac{y^{2^{k}(1+q)}}{2^{1+\cdots+2^{k}}}+\sum_{i=1}^{n-1} \sum_{k=n-i-1}^{n-2} \frac{\rho_{i}^{2^{k}}}{2^{1+\cdots+2^{k}}} y^{2^{k}\left(1+2^{i}\right)}=1+\tilde{u},
$$

and one checks that $v(\tilde{u})=v\left(y^{q / 2}\right)$. From the equality

$$
f_{\rho}(y) u^{2}-1=\sum_{k=0}^{n-1} \rho_{k} y^{1+2^{k}}+y^{q}+y^{1+q}+(1+T) 2 \tilde{u}+(1+T) \tilde{u}^{2},
$$

taking into account that $v_{L}(2 T \tilde{u})>s, v_{L}\left(T \tilde{u}^{2}\right)>s, \forall 0 \leq k \leq n-2$, $v_{L}\left(\rho_{k} y^{1+2^{k}}\right)>s$ and expanding $\tilde{u}$ and $\tilde{u}^{2}$ one gets modulo $\pi_{L}^{s} \mathfrak{m}$

$$
\begin{aligned}
f_{\rho}(y) u^{2}-1 \equiv & \rho_{n-1} y^{1+q / 2}-2 y^{q / 2}+2 y^{q}-\sum_{k=1}^{n-2} \frac{2 y^{2^{k}(1+q)}}{2^{1+\cdots+2^{k}}}+\sum_{k=1}^{n-1} \frac{y^{2^{k}(1+q)}}{2^{2+\cdots+2^{k}}}+ \\
& +\sum_{i=1}^{n-1} \sum_{k=n-i-1}^{n-2} \frac{2 \rho_{i}^{2^{k}} y^{2^{k}\left(1+2^{i}\right)}}{2^{1+\cdots+2^{k}}}+\sum_{i=1}^{n-1} \sum_{k=n-i}^{n-1} \frac{\rho_{i}^{2^{k}} y^{2^{k}\left(1+2^{i}\right)}}{2^{2+\cdots+2^{k}}} .
\end{aligned}
$$

Arranging the terms of (24), taking into account that $v_{L}\left(2 y^{q}\right)>s$ and for all $2 \leq i \leq n-1$ and $n-i \leq k \leq n-2$

$$
v_{L}\left(\rho_{i}^{2^{k}} y^{2^{k}\left(1+2^{i}\right)} \frac{2}{2^{2+\cdots+2^{k}}}\right)>s
$$

and comparing with (23) , one obtains $f_{\rho}(y) u^{2}-1 \equiv \rho_{n-1} y^{1+q / 2}+2 h y^{q / 2} \bmod \pi_{L}^{s} \mathfrak{m}$.

Step F: The ramification filtration of $M / L$ is

$$
H_{0}=H_{1}=\cdots=H_{1+q} \supsetneq\{1\} .
$$

One has to show that $v_{M}\left(\mathcal{D}_{M / L}\right)=q+2$, we will use freely results from Ser79] IV. If $\rho_{n-1}=0$, then according to Step E, one has

$$
f_{\rho}(y) u^{2}=1+2 y^{q / 2} h+r,
$$

and one concludes using [CM11] Lemma 2.1. Else, if $\rho_{n-1} \neq 0$, one has

$$
\max _{u \in L^{\times}} v_{L}\left(f_{\rho}(y) u^{2}-1\right) \geq v_{L}\left(\rho_{n-1} y^{1+q / 2}\right),
$$

then [LRS93] Lemma 6.3 implies that $v_{M}\left(\mathcal{D}_{M / L}\right) \leq q+3$. Using Step B, Step D and [Ser79] IV $\S 2$ Proposition 11, one has that the break in the ramification filtration of $M / L$ is congruent to $1 \bmod 2$, i.e. $v_{M}\left(\mathcal{D}_{M / L}\right) \leq q+2$. 
According to Step $\mathbf{D}$ and lemma 2.1, the break $t$ of $M / L$ is in $1+q \mathbb{N}$. If $t=1$ then $G_{2}=\{1\}$ and $G_{1} / G_{2}=G / G_{2} \simeq G$ would be abelian, so $t \geq 1+q$, i.e. $v_{M}\left(\mathcal{D}_{M / L}\right) \geq q+2$.

Step G : Computations of conductors.

For $l \neq 2$ a prime number, the $G$-modules $\operatorname{Jac}(C)[l]$ and $\operatorname{Jac}\left(\mathcal{C}_{k}\right)[l]$ being isomorphic one has that for $i \geq 0$ :

$$
\operatorname{dim}_{\mathbb{F}_{l}} \operatorname{Jac}(C)[l]^{G_{i}}=\operatorname{dim}_{\mathbb{F}_{l}} \operatorname{Jac}\left(\mathcal{C}_{k}\right)[l]^{G_{i}} .
$$

Moreover, for $0 \leq i \leq 1+q$ one has $\operatorname{Jac}\left(\mathcal{C}_{k}\right)[l]^{G_{i}} \subseteq \operatorname{Jac}\left(\mathcal{C}_{k}\right)[l]^{\mathrm{Z}(G)}$, then from $\mathcal{C}_{k} / \mathrm{Z}(G) \simeq \mathbb{P}_{k}^{1}$ and lemma 2.2 it follows that for $0 \leq i \leq 1+q$, $\operatorname{dim}_{\mathbb{F}_{l}} \operatorname{Jac}\left(\mathcal{C}_{k}\right)[l]^{G_{i}}=0$. Since $g(C)=q / 2$ one gets $f(\operatorname{Jac}(C) / K)=2 q+1$ and $\operatorname{sw}\left(\operatorname{Jac}(C) / \mathbb{Q}_{2}^{\text {ur }}\right)=1$.

Example : Magma codes are available on the author webpage. Let $K:=\mathbb{Q}_{2}^{\text {ur }}\left(2^{1 / 5}\right)$ and $f(X):=1+2^{6 / 5} X^{2}+2^{4 / 5} X^{3}+X^{4}+X^{5} \in K[X]$, one checks that the smooth, projective, integral curve birationally given by $Y^{2}=f(X)$ has the announced properties, that is the wild monodromy $M / K$ has degree 32 and one can describe its ramification filtration. The first program checks that Step A and Step D hold for this example. The second program checks Step $\mathbf{F}$ and is due to Guardia, J., Montes, J. and Nart, E. (see [GMN11]) and computes $v_{M}\left(\mathcal{D}_{M / \mathbb{Q}_{2}^{\text {ur }}}\right)=194$. Using [Ser79] III $\S 4$ Proposition 8 , one finds that $v_{M}\left(\mathcal{D}_{M / K}\right)=66$, which was the announced result in Theorem 3.13.

\section{Remarks :}

1. The above example was the main motivation for Step $\mathbf{F}$ since it shows that one could expect the correct behaviour for the ramification filtration of $\operatorname{Gal}(M / K)$ when $p=2$.

2. The naive method to compute the ramification filtration of $M / K$ in the above example fails. Indeed, in this case Magma needs a huge precision when dealing with 2-adic expansions to get the correct discriminant.

Acknowledgements : I would like to thank M. Monge for pointing out lemma 2.1.

\section{References}

[BK05] A. Brumer and K. Kramer. The conductor of an abelian variety. Compositio mathematica, n 2(92), 2005. 
[CM11] P. Chrétien and M. Matignon. Maximal monodromy in unequal characteristic. submitted, 2011.

[GMN11] J. Guardia, J. Montes, and E. Nart. Higher Newton polygons in the computation of discriminants and prime ideal decomposition in number fields. Journal de théorie des nombres de Bordeaux, 2, 2011.

[Gur03] R. Guralnick. Monodromy groups of coverings of curves. In Galois groups and fundamental groups, volume 41. MSRI Publications, 2003.

[Hup67] B. Huppert. Endliche Gruppen I. Grundlehren der Mathematischen Wissenschaften, (134), 1967.

[Kra90] A. Kraus. Sur le défaut de semi-stabilité des courbes elliptiques à réduction additive. Manuscripta Mathematica, (69), 1990.

[Liu02] Q. Liu. Algebraic Geometry and Arithmetic Curves. Oxford University Press, 2002.

[LM05] C. Lehr and M. Matignon. Automorphism groups for p-cyclic covers of the affine line. Compositio Mathematica, n 5(141), 2005.

[LRS93] P. Lockhart, M.I. Rosen, and J. Silverman. An upper bound for the conductor of an abelian variety. Journal of algebraic geometry, n 2, 1993.

[Ogg67] A.P. Ogg. Elliptic curves and wild ramification. American Journal of Mathematics, 89(1), 1967.

[Ray90] M. Raynaud. p-groupes et réduction semi-stable des courbes. In Birkhäuser, editor, The Grothendieck Festschrift, Vol. III, 1990.

[Ser67] J.-P. Serre. Représentations linéaires des groupes finis. Hermann, Paris, 1967.

[Ser79] J.-P. Serre. Local Fields. Graudate Texts in Mathematics (67), 1979.

[ST68] J.-P. Serre and J. Tate. Good reduction of abelian varieties. Annals of Mathematics, (88), 1968.

[Suz86] M. Suzuki. Group Theory II. Grundlehren der Mathematischen Wissenschaft (248), 1986. 